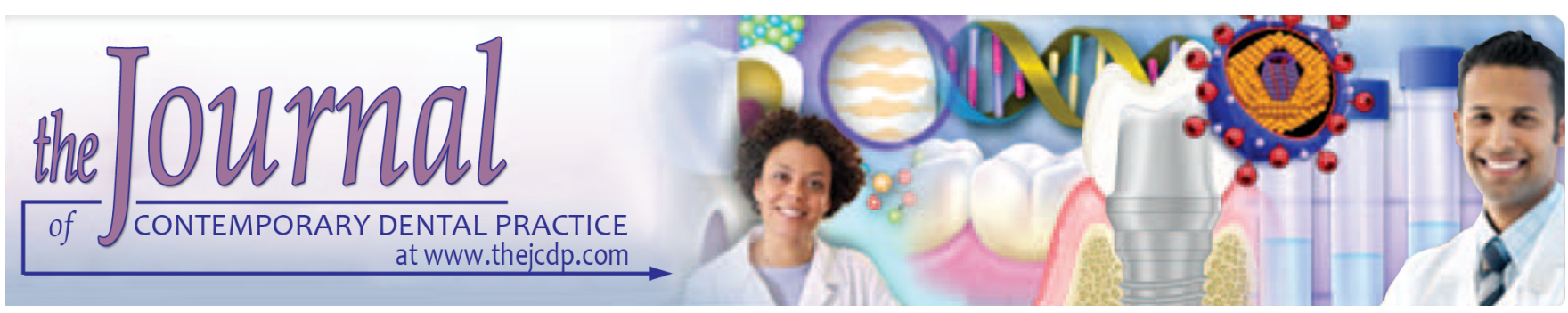

\title{
Prophylactic and Therapeutic Antibiotic Patterns of Lebanese Dentists for the Management of Dentoalveolar Abscesses
}

${ }^{1}$ Ghada Asmar, ${ }^{2}$ Dominique Cochelard, ${ }^{3}$ Jacques Mokhbat, ${ }^{4}$ Mohamed Lemdani, ${ }^{5}$ Ahmed Haddadi, ${ }^{6}$ Fouad Ayoub

\begin{abstract}
Introduction: General dentists issue approximately $10 \%$ of antibiotic prescriptions across the global medical community consummation. The use of antibiotics for the management of dentoalveolar infections should be considered only in the presence of an increased risk of a systemic involvement or to prevent metastatic infections. This study aimed to investigate the prophylactic and therapeutic antibiotic prescription patterns of Lebanese dentists for the management of dentoalveolar abscesses. The aim was to evaluate the influence of the patients' medical condition and clinical signs data on the patterns of antibiotics prescription.
\end{abstract}

Materials and methods: Only patients with a dentoalveolar abscess were included in the study. Age, medical history, reason for consultation, clinical signs and symptoms, diagnosis, type of local treatment, and type of antibiotherapy were collected for each patient attending dental clinics in Beirut. The data were analyzed with chi-square test and multivariate regression.

Results: Out of the 563 initial patients, 127 were selected for the study and received a local treatment. The patient's medical condition and age did not affect the decision to prescribe antibiotics $36.2 \%$ patients with pain and $11.8 \%$ patients with swelling were prescribed antibiotics. Pain and swelling

${ }^{1}$ Doctorate School for Science and Technology, Research Platform for Environmental Sciences, Faculty of Dental Medicine, Lebanese University, Beirut, Lebanon

${ }^{2,4}$ Biomathematics Laboratory EA 2694, Lille 2 University, Lille France

${ }^{3}$ Division of Infectious Diseases, School of Medicine, Lebanese American University, Beirut, Lebanon

${ }^{5}$ Epidemiology and Quality of Care, Lille 2 University, Lille France

${ }^{6}$ Department of Basic Sciences, Faculty of Dental Medicine Lebanese University, Beirut, Lebanon

Corresponding Author: Ghada Asmar, PhD Student Doctorate School for Science and Technology, Research Platform for Environmental Sciences, Faculty of Dental Medicine, Lebanese University, Beirut, Lebanon, Phone: +9613234189, e-mail: ghadaasmar@gmail.com contributed to a higher level of antibiotic prescription compared to other signs and symptoms. Antibiotics were prescribed inappropriately to 51.76 and $38.10 \%$ among patients with an acute or chronic dentoalveolar abscess respectively. The main prescribed antibiotic was amoxicillin.

Conclusion: This study showed that dentists often did not follow the current prophylactic and therapeutic antibiotic prescription guidelines.

Clinical significance: Antibiotics prescriptions in dentistry will be more pertinent, leading to a decrease in inadequacy of prescriptions, microbial resistance, and the development of multiresistant germs against antibiotics.

Keywords: Acute abscess, Antibiotics, Cellulitis, Dental infection, Prescriptions.

How to cite this article: Asmar G, Cochelard D, Mokhbat J, Lemdani M, Haddadi A, Ayoub F. Prophylactic and Therapeutic Antibiotic Patterns of Lebanese Dentists for the Management of Dentoalveolar Abscesses. J Contemp Dent Pract 2016;17(6):425-433.

Source of support: Nil

Conflict of interest: None

\section{INTRODUCTION}

The use of antibiotics for the management of dentoalveolar infections should be considered only in the presence of an increased risk of a systemic involvement. ${ }^{1-3}$ They are also prescribed prophylactically, according to specific guidelines, to prevent metastatic infections. ${ }^{4-6}$ However, antibiotics have been prescribed empirically and systemically leading to an increased microbial resistance. ${ }^{7,8}$

General dentists issue approximately $10 \%$ of antibiotic prescriptions across the global medical community consummation. ${ }^{9,10}$ Several studies, conducted in different geographical areas, have investigated the antibiotic prescribing protocols of dentists in relation to the patient's medical condition, and the presence of swelling and pain. ${ }^{7-11}$

The therapeutic and prophylactic guidelines of antibiotics prescriptions in dentistry differ among the international 
recommendations of health authorities. ${ }^{5}$ In Lebanon, a very important part of practicing dentists have graduated from foreign universities (European Union, North America, Asia, and few of them graduated from Brazil and North Africa) and so have been exposed to different antibiotic prescription guidelines for prophylactic and therapeutic purposes. This can result in confusion among the prescribing dentists, especially in the absence of clear directives from the local medical and dental authorities.

The objective of this study was to investigate the patterns of prophylactic and therapeutic prescription of antibiotics followed by Lebanese dentists in the management of patients with dentoalveolar infections.

\section{MATERIALS AND METHODS}

\section{Ethical Approval}

This study concerns prospective and noninterventional data patient. All patients were informed about the aim of the study and written consent was obtained from each participant. This study was approved by the Institutional Review Board and Ethical Committee of the Lebanese University.

\section{Study Design}

This prospective multicenter study was conducted over a period of 9 months. It involved patients who sought a consultation and/or a treatment from dentists at the Faculty of Dentistry, Lebanese University, and private dentists practicing in the Greater Area of Beirut. Fiftyfive dentists participated in the study. Only patients at least 16 years old and presenting with a dentoalveolar abscess were included in the study. Patients who needed hospitalization and for whom data was missing were systematically excluded from the study.

The following information were collected using a standardized form (Appendix), including age, reason for consultation, medical history (severity of underlying disease according to McCabe score), clinical signs and symptoms, clinical diagnosis, treatment provided, and type, dosage, and duration of prophylactic, and therapeutic antibiotics.

The McCabe score is used as follows: Score 0 for patients with no underlying disease, and score 1 for patients with a nonfatal underlying disease or disease estimated to become fatal in at least 5 years. ${ }^{12}$

The outcome of the study: The rate of inadequate prophylactic and curative antibiotic prescriptions decreased.

\section{Statistical Analysis}

The collected data was analyzed using the statistical software [Statistical Package for the Social Sciences (SPSS) 19.0, USA]. To determine whether there is a significant association between two categorical variables from the same population, dependence between the prophylactic prescriptions, McCabe score, and age on one side, and dependence between the therapeutic prescriptions, McCabe score, age, clinical diagnosis (acute or chronic dentoalveolar abscess), and reason of consultation (pain and/or swelling) on the other side were tested with chisquare tests. Fisher's exact test is used when the count is less than 5 . The level of significance was set to $p<0.05$. A backward logistic regression was performed to evaluate the impact of the independent variables (such as McCabe score, pain, localized swelling, diffuse swelling, swelling involving the vestibule, presence of pus, and discomfort to percussion) for antibiotics prescriptions.

\section{RESULTS}

During the study period, 741 patients received a consultation at different clinics. Five hundred sixty-three (563) patients were at least 16 years old. Four hundred thirty-six of those did not present with a dentoalveolar abscess, and therefore were excluded from the study. In total, 127 patients, 72 males and 55 females, were selected for the study (Flow Chart 1).

The gender ratio was 1:3 (56.7\% male and $43.3 \%$ female). The mean age was $43.5 \pm 14.9$ years (16-75), 6 patients being more than 60 years old. The median was 45 and interquartile range was 26.

McCabe score 1 was attributed to 32 patients (25.20\%). Ninety-five patients $(74.80 \%)$ did not have any underlying disease and were attributed a score 0 . Table 1 shows the different types of underlying diseases for score 1 patients and the frequency of prophylactic and therapeutic prescriptions respectively. The $\mathrm{McCabe}$ score and age of the patients did not affect the incidence of prophylactic ( $p=0.303$ and $p=0.417$ respectively) and therapeutic ( $p=0.084$ and $p=0.823$ respectively) prescriptions. A multivariable regression revealed that the presence of an underlying disease predisposed to prescribing therapeutic antibiotics $(\mathrm{p}=0.014)$.

Flow Chart 1: Scheme of the study model

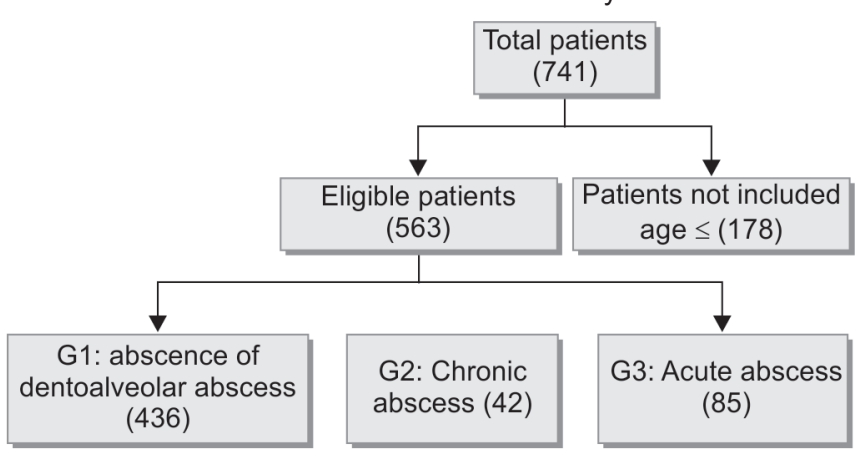

G1: Group of patients excluded because of absence of dentoalveolar abscess; G2: Group of patients included with chronic abscess; and G3: Group of patients included with acute abscess 
Prophylactic and Therapeutic Antibiotic Patterns of Lebanese Dentists for the Management of Dentoalveolar Abscesses

Table 1: Underlying disease with prophylactic/nonprophylactic prescription and curative/noncurative prescription

\begin{tabular}{|c|c|c|c|c|}
\hline Underlying disease & $\begin{array}{l}\text { Prophylactic } \\
\text { prescription, } n(\%)\end{array}$ & $\begin{array}{l}\text { No prophylactic } \\
\text { prescription, } n(\%)\end{array}$ & $\begin{array}{l}\text { Therapeutic } \\
\text { prescription, } n(\%)\end{array}$ & $\begin{array}{l}\text { No therapeutic } \\
\text { prescription, } n(\%)\end{array}$ \\
\hline Stent & & $4(12.50)$ & $4(12.50)$ & \\
\hline $\mathrm{AHT}^{*}$ & & $5(15.63)$ & $1(3.13)$ & $4(12.50)$ \\
\hline $\mathrm{AHT}+$ overweight & & $2(6.25)$ & $2(6.25)$ & \\
\hline $\mathrm{AHT}+$ tobacco & $1(3.13)$ & & $1(3.13)$ & \\
\hline $\mathrm{AHT}+$ tobacco + overweight & & $1(3.13)$ & & $1(3.13)$ \\
\hline $\mathrm{AHT}+$ diabetes + overweight & $1(3.13)$ & & $1(3.13)$ & \\
\hline $\mathrm{AHT}+$ diabetes & & $1(3.13)$ & $1(3.13)$ & \\
\hline Diabetes + tobacco & & $1(3.13)$ & $1(3.13)$ & \\
\hline Diabetes & & $1(3.13)$ & $1(3.13)$ & \\
\hline Allergy & $1(3.13)$ & $3(9.38)$ & $4(12.50)$ & \\
\hline Allergy + asthma + corticotherapy & & $2(6.25)$ & & $2(6.25)$ \\
\hline Anti-depressive & & $1(3.13)$ & $1(3.13)$ & \\
\hline Tobacco & & $2(6.25)$ & $2(6.25)$ & \\
\hline Overweight & & $1(3.13)$ & & $1(3.13)$ \\
\hline Cholesterol & & $1(3.13)$ & $1(3.13)$ & \\
\hline Multiple sclerosis & & $1(3.13)$ & $1(3.13)$ & \\
\hline Prosthetic cardiac valve + stent + anti-depressive & $2(6.25)$ & & $2(6.25)$ & \\
\hline Cancer $>5$ years & $1(3.13)$ & & $1(3.13)$ & \\
\hline Total & $6(18.75)$ & $26(81.25)$ & $24(75.00)$ & $8(25)$ \\
\hline $\mathrm{p}$-value for McCabe score & $p=0.303$ & & $p=0.084$ & \\
\hline$p$-value for age & $p=0.417$ & & $p=0.823$ & \\
\hline
\end{tabular}

${ }^{*} \mathrm{AHT}$ : Arterial hypertension

Table 2: Number (percentage) of therapeutic prescriptions by reason of consultation

\begin{tabular}{lllll}
\hline & \multicolumn{2}{c}{ Therapeutic prescriptions } & & \\
\cline { 2 - 3 } Reason for consultation & No $n(\%)$ & Yes $n(\%)$ & $20(15.74)$ & $p$-value \\
\hline Ordinary consultation & $16(33.34)$ & $4(5.08)$ & $15(11.81)$ & 0.001 \\
Edema & $7(14.58)$ & $8(10.12)$ & $46(36.22)$ & 0.027 \\
Pain & $15(31.25)$ & $31(39.24)$ & $46(36.22)$ & \\
Pain and edema & $10(20.83)$ & $36(45.56)$ & $127(100)$ & \\
Total & $48(100)$ & $79(100)$ & & \\
\hline
\end{tabular}

${ }^{*} p$-value $\leq 0.05$

Table 3: Number (percentage) of curative prescriptions by type of abscess

\begin{tabular}{|c|c|c|c|}
\hline & $\begin{array}{l}\text { Acute abscess* } \\
\text { (85/127 patients) }\end{array}$ & $\begin{array}{l}\text { Chronic abscess }^{\dagger} \\
\text { (42/127 patients) }\end{array}$ & $\begin{array}{l}\text { Total }(127 / 127 \\
\text { patients) }\end{array}$ \\
\hline $\begin{array}{l}\text { Therapeutic } \\
\text { prescription }\end{array}$ & $63 / 127(74.1 \%)$ & 16/127 (38.1\%) & $\begin{array}{l}79 / 127 \\
(62.2 \%)\end{array}$ \\
\hline
\end{tabular}

*A therapeutic prescription was not given for 22 patients with an acute abscess; ${ }^{\dagger} A$ therapeutic prescription was not given for 26 patients with a chronic dental abscess

The different reasons for consultation are described in Table 2. The percentage of patients who received therapeutic prescriptions is reported in Table 3 . The levels of therapeutic prescriptions were higher in the presence of pain $(\mathrm{p}=0.001)$ and swelling $(\mathrm{p}=0.027)$.

Moreover, 85 (66.90\%) and $42(33.10 \%)$ patients presented with acute and chronic dentoalveolar abscesses respectively. Table 3 shows the distribution of therapeutic prescriptions for the two types of dentoalveolar abscesses.
Thirty-eight patients underwent incision and drainage; 30 patients had their tooth extracted; and endodontic treatments were provided to 59 patients.

Sixty-three patients with an acute dentoalveolar abscess were given a therapeutic prescription. Nineteen of those had aggravating signs and symptoms (Table 4). Chi-square/exact Fisher independence tests showed no significant relation between the prescription of antibiotics and any of the following aggravating symptoms or signs: Lymphatic adenopathy $(\mathrm{p}=0.622)$, trismus $(\mathrm{p}=0.615)$, fever $(p=0.681)$, diffuse swelling $(p=0.340)$, and swelling in the vestibule $(\mathrm{p}=0.926)$.

Multivariable regression showed that underlying disease (McCabe score $1 ; \mathrm{p}=0.014)$, pain $(\mathrm{p}=0.001)$, localized swelling $(p=0.013)$, and pus $(p=0.02)$ were predisposing factors to prescribing therapeutic antibiotics (Table 5). The odds ratio determines and compares the magnitude of various risk factors for the curative prescription. The Hosmer-Lemeshow test shows that the model fits well with the data $(p=0.987)$. 
Table 4: Clinical signs of acute abscess cases where curative antibiotics were prescribed

\begin{tabular}{|c|c|c|c|}
\hline Abscess & Clinical signs & $n(\%)^{*}$ & $p$-value ${ }^{\dagger}$ \\
\hline $\begin{array}{l}\text { Acute abscess } \\
\text { without } \\
\text { aggravating } \\
\text { clinical signs } \\
(n=44)\end{array}$ & $\begin{array}{l}\text { Pain } \\
\text { Pain at palpation } \\
\text { Pain at percussion } \\
\text { Pus } \\
\text { Localized swelling }\end{array}$ & $\begin{array}{l}37(84.09) \\
1(2.27) \\
23(52.27) \\
32(72.72) \\
22(50)\end{array}$ & \\
\hline $\begin{array}{l}\text { Acute abscess } \\
\text { with aggravating }\end{array}$ & $\begin{array}{l}\text { Swelling in the sulcus } \\
\text { of the vestibule }\end{array}$ & $7(36.84)$ & 0.926 \\
\hline clinical signs & Fever & $2(10.52)$ & 0.681 \\
\hline \multirow[t]{4}{*}{$(n=19)$} & $\begin{array}{l}\text { Diffused edema } \\
\text { Periorbital diffused } \\
\text { edema }\end{array}$ & $\begin{array}{l}13(68.42) \\
0(0)\end{array}$ & 0.34 \\
\hline & Trismus & $1(5.26)$ & 0.615 \\
\hline & $\begin{array}{l}\text { Difficulty at } \\
\text { swallowing }\end{array}$ & $0(0)$ & \\
\hline & $\begin{array}{l}\text { Significant lymphatic } \\
\text { adenopathy }\end{array}$ & $1(5.26)$ & 0.622 \\
\hline
\end{tabular}

${ }^{\dagger} p$-value $\leq 0.05$; *A total of 85 patients presented with an acute abscess

Different types of antibiotics were prescribed prophylactically and therapeutically (Table 6) according to different regimens, dosage, and duration (Table 7).

\section{DISCUSSION}

The management of acute dentoalveolar abscesses is based on the principle of drainage achieved either

Table 6: Different types of antibiotics prescribed prophylactically and therapeutically

\begin{tabular}{lll}
\hline Prescription & Antibiotics & $n(\%)$ \\
\hline Prophylactic & Amoxicillin & $2(11.8)$ \\
antibiotherapy & Amoxicillin + clavulanic acid & $15(88.2)$ \\
& Total & $17(100)$ \\
Therapeutic & Amoxicillin & $3(3.8)$ \\
antibiotherapy & Amoxicillin + clavulanic acid & $51(64.6)$ \\
& Amoxicillin + metronidazole & $2(2.5)$ \\
& Levofloxacin & $2(2.5)$ \\
& Spiramycin + metronidazole & $21(26.6)$ \\
& Total & $79(100)$ \\
\hline
\end{tabular}

Table 5: p-values for curative prescriptions with multivariate logistic regression (backward stepwise)

\begin{tabular}{lll}
\hline Independent variables & \multicolumn{1}{c}{$\begin{array}{l}\text { Odds ratio } \\
\text { adjusted }\end{array}$} \\
\hline Age & $\begin{array}{l}0.592,0.419, \\
\text { and } 0.308\end{array}$ & \\
McCabe score & 0.014 & 4.022 \\
Edema (reason for consultation) & 0.900 & \\
Pain (reason for consultation) & 0.001 & 5.863 \\
Pain at percussion & 0.238 & \\
Localized swelling & 0.013 & 4.026 \\
Diffuse swelling & 0.270 & \\
Swelling in the sulcus of the & 0.117 & \\
vestibule & & \\
Pus & 0.020 & 2.9 \\
\hline
\end{tabular}

${ }^{*} p$-value: $p \leq 0.05 ; p$-value for the test of Hosmer and Lemeshow is significant: $p$-value $=0.987>0.02$, the model fits well with the data

endodontically or surgically. Antibiotics are indicated only in the presence of aggravating signs or symptoms, such as diffuse swelling, trismus, fever, and lymphadenopathy. ${ }^{13}$ Antibiotics are prescribed empirically ${ }^{7,8}$ and systemically with disregard to the international guidelines, ${ }^{4-6}$ which indicate their use in specific cases, such as immunosuppressant or radiation therapy of the ore-facial sphere, or in the presence of an increased risk of infection propagation and systemic involvement.

Several studies have evaluated the attitude of dentists with regards to the management of dental infections. ${ }^{7,8,11,13}$ The present study evaluated the management of chronic and acute dentoalveolar abscesses by Lebanese dentists. This study focused mainly on the prophylactic and therapeutic prescription of antibiotics based on patients' health, age, and clinical examination.

Age, medical history and underlying disease, and prescribed drugs are important parameters to be considered during the evaluation of a patient's medical

Table 7: Therapeutic and prophylactic prescription

\begin{tabular}{|c|c|c|c|c|c|c|c|c|c|c|c|}
\hline \multirow[b]{2}{*}{ Prescription } & \multirow[b]{2}{*}{ Antibiotic } & \multirow[b]{2}{*}{ Dosage/day } & \multicolumn{9}{|c|}{ Duration/day } \\
\hline & & & 1 & 2 & 3 & 4 & 5 & 6 & 7 & 8 & 10 \\
\hline \multirow[t]{2}{*}{ Prophylactic prescription } & Amoxicillin & $2 \mathrm{gm}$ & & & & & 1 & & 1 & & \\
\hline & Amoxicillin/clavulanic acid & $2 \mathrm{gm}$ & 3 & 1 & 4 & & 3 & & 3 & & 1 \\
\hline \multirow[t]{9}{*}{ Therapeutic prescription } & Amoxicillin & $2 \mathrm{gm}$ & & 1 & & & 1 & & 1 & & \\
\hline & Amoxicillin/clavulanic acid & $1.875 \mathrm{gm}$ & & & & & 1 & & & & \\
\hline & Amoxicillin/clavulanic acid & $2 \mathrm{gm}$ & & & 2 & 4 & 4 & 5 & 34 & 1 & \\
\hline & Amoxicillin + metronidazole & 1 and $1 \mathrm{gm}$ & & & & & 1 & & & & \\
\hline & $\begin{array}{l}\text { Amoxicillin + metronidazole } \\
\text { levofloxacin }\end{array}$ & 2 and $1 \mathrm{gm}$ & & & & & & 1 & & & \\
\hline & Spiramycin + metronidazole & & & & & & & & 1 & & \\
\hline & Spiramycin + metronidazole & 0.75 and $0.375 \mathrm{gm}$ & & & & & 1 & 1 & 14 & & \\
\hline & Spiramycin + metronidazole & 2 and $1 \mathrm{gm}$ & & & & & & 1 & & & \\
\hline & Spiramycin + metronidazole & 4.5 and $0.75 \mathrm{gm}$ & & & & & & & 2 & & \\
\hline
\end{tabular}


history. For this reason, these factors were included in the evaluation of antibiotics prescriptions, prophylactically or therapeutically, by Lebanese dentists.

A patient's medical condition can be reflected by the McCabe score. This score indicates the duration of survival in the presence of an underlying disease (when the disease becomes fatal): Less than 1 year (score 3), between 1 and 5 years (score 2), more than 5 years, or if the disease is nonfatal (score 1). Score 0 is attributed to patients without any disease.

To the authors' knowledge, in outpatient dentistry, this is the first study to evaluate the relation between the McCabe score and the prescription of antibiotics for the management of acute dentoalveolar abscesses.

The patients had a McCabe score $0(74.80 \%)$ or $1(25.20 \%)$. Patients with higher scores would not be expected to seek dental treatment in a dental clinic but would rather be hospitalized. Previously, the patient's medical status seemed to influence the decision to prescribe antibiotics ${ }^{11}$ with a wide variation in the dosage and duration. ${ }^{7}$ These findings were confirmed in the present study.

There was no relation between the patient's medical status and the prescription of prophylactic and therapeutic antibiotics prescribed by Lebanese dentists. However, combined with other parameters (pain, pain to percussion, localized and diffuses swelling, pus discharge, swelling in the sulcus of the vestibule), the McCabe score appeared to be a criterion favoring the prescription of therapeutic antibiotics: $75 \%$ of the patients with a McCabe score 1 were prescribed antibiotics for therapeutic purposes. Direct comparison with the results of Tulip and Palmer ${ }^{11}$ could not be made due to differences in the methodology. Interestingly, 1,472 patients were included in their research. The relatively moderate group size of the present study (127 patients) may have resulted in a loss of statistical power, which in turn could explain the absence of relation between the patient's medical gravity assessed by McCabe score and the prescription of antibiotics.

Only two patients had a high risk of underlying disease (prosthetic valve). Prophylactic antibiotics were prescribed to these patients. The British Cardiac Society (BCS) and the American Heart Association (AHA) recommend the prescription of antibiotics for those patients before specific dental treatments to prevent infective endocarditis. ${ }^{5}$ On contrary, antibiotic prophylaxis is not required according to the National Institute for Health and Clinical Excellence guidelines. ${ }^{14}$ Moreover, the AHA and the BCS have different guidelines in this context with respect to the cardiac conditions for which antibiotic prophylaxis is recommended. Obviously, the need for internationally approved guidelines is of utmost importance.
Prophylactic antibiotics were inappropriately prescribed to $11.6 \%$ of the patients without an underlying disease (McCabe score 0 ). Similarly, some patients with a McCabe score 1 not necessitating prophylaxis were given a prescription. In addition, variability was observed among dentists regarding antibiotic prophylaxis for a specific underlying disease, such as allergy and diabetes. These findings indicate a lack of knowledge and a misunderstanding of the involved pathologies, the impairment of the host defenses, the indication of prophylactic antibiotherapy, and the need to educate dentists on current prescription guidelines to favorably change their prescription behavior. ${ }^{15}$

However, age is an important factor to consider because the complications associated with adverse effects resulting from the use of antibiotics can be more serious in very young and old patients. At least two previous studies reported that antibiotics were prescribed inappropriately to children most probably in a preventative proactive manner. ${ }^{11,16}$ Considering that many unwarranted prescriptions were based on different medical conditions, it was expected that similar unnecessary prescriptions would be given to older patients on the assumption that these individuals, similar to the very young ones, would be more susceptible to the occurrence of infection. However, it appeared that age was not a factor on which dentists based their decision to prescribe antibiotics prophylactically or therapeutically. Interestingly, age was not evaluated as an influencing factor in any of the previous studies related to the prescription of antibiotics for the management of dental infections.

Previous studies showed that about 75 and $13 \%$ of the patients presented with pain or swelling respectively. ${ }^{11,16}$ Pain and swelling occurred simultaneously in $6.25 \%$ of the patients. ${ }^{11}$ Those studies evaluated more than 1,000 patients. In the present study involving 127 patients, those percentages were $36.2,11.8$, and $36.2 \%$ for pain, swelling, and both concurrently (Table 2). The large differences for pain, and pain and swelling were probably related to the difference in size between the evaluated groups.

The influence of the reason for consultation on the decision to prescribe antibiotics has already been evaluated. ${ }^{16,17}$ Dailey and Martin ${ }^{16}$ found that $62 \%$ of adult patients with pain and/or swelling did not receive any treatment; in another study, ${ }^{18} 59 \%$ of the patients with periapical abscesses were given antibiotics only, without any local treatment. If treatments were rendered to those patients, the number of inappropriate prescriptions in these studies could be lower. The absence of treatment was attributed to the lack of time, incorrect diagnosis, and patient's expectations. On contrary, $100 \%$ of the patients in this study were provided a local treatment. The differences among the studies could be related to the groups' 
respective sizes. A larger group in this study could result in patients not receiving a local treatment. In a study by Palmer et al, ${ }^{7}$ the local treatment option was discussed with only $16.2 \%$ of the patients.

The prescription of therapeutic antibiotics should be limited to patients with signs of systemic involvement, such as fever, fatigue, lymphadenopathy, ${ }_{1}^{17,19}$ and diffuse swelling. Eighty-five patients presented with signs and symptoms of an acute dentoalveolar abscess as defined by the American Association of Endodontists. ${ }^{20}$ Twentytwo patients did not receive any antibiotics. Sixty-three patients were given therapeutic antibiotics (Table 4). The prescription of antibiotics based on clinical signs or symptoms was not justified for $51.8 \%(44 / 85)$ of these patients presenting with either pain or a localized swelling. Multivariable regression (Table 5) showed that the presence of pain or a localized swelling contributed to the decision to prescribe antibiotics and confirmed the results of previous studies. ${ }^{7,8,11}$ An inappropriate prescription related to the presence of a localized swelling was given to $22 / 85$ patients $(25.9 \%)$. Similar results were found in previous studies. ${ }^{78,15}$ These results were not surprising because it was previously observed that dentists were reticent on prescribing antibiotics for the management of localized swellings: Despite a course given to dentists on the current prescribing guidelines, the level of unjustified prescriptions after the course increased in the presence of a localized swelling. ${ }^{15}$ In this study, a proper decision with regards to prescribing antibiotics or refraining from prescribing them was made for 19 and 22 patients respectively, corresponding to $48.3 \%$ of patients with an acute dentoalveolar abscess. Within this context, antibiotics were dispensed inappropriately to $16 / 42$ patients $(38.1 \%)$ with a chronic dentoalveolar abscess; antibiotics were not administered rightfully to the $26 / 42$ remaining patients with a chronic abscess. A proper decision, to prescribe or to refrain from prescribing antibiotics, was made for $67 / 127$ patients $(52.8 \%)$. The level of inappropriate decisions was slightly lower in this study compared to the results of Tulip and Palmer; ${ }^{11}$ they found that about $50 \%$ of the patients received antibiotics without treatment. However, this difference could be related to the size of the samples in the two studies. Another explanation could be the access to a local treatment to all of the patients in this study; whereas, a local treatment was discussed with only $16.2 \%$ of the patients in the study of Tulip and Palmer. ${ }^{11}$

The current recommendations for antibiotic prophylaxis vary among the countries. ${ }^{5}$ Amoxicillin is the antibiotic of choice. ${ }^{5,6}$ However, it was alarming to observe that Amoxicillin combined with clavulanic acid was prescribed to $88.20 \%$ of the patients in this study. Also, Lebanese dentists did not follow the current guidelines ( $2 \mathrm{gm} 1$ hour prior to treatment) pertaining to the dosage, duration, and frequency. These improper patterns of prescriptions would contribute to the emergence of microbial resistance and a higher occurrence of antibiotic allergies. ${ }^{16,21}$ Continuous educations could help in changing the behavior of dentists in prescribing prophylactic antibiotics. ${ }^{15}$

The current recommendations for therapeutic antibiotics also vary among countries. ${ }^{6,22}$ In North America, Penicillin $\mathrm{V}$ and $\mathrm{K}$ are the antibiotics of choice for the management of dental infections. Clindamycin is a first choice antibiotic in case of allergy to penicillin. ${ }^{5,6}$ Amoxicillin and metronidazole are second choice antibiotics. ${ }^{6}$ For West European countries (England, France, and Belgium) amoxicillin is the first choice. ${ }^{5,6}$ None of the dentists involved in the current study prescribed penicillin. This finding could be related to the European influence in the dental education of the Lebanese dentists. Amoxicillin with or without clavulanic acid was the most administered antibiotic confirming the results from other studies conducted in different countries. ${ }^{7-9}$ In contrast, narrow spectrum antibiotics were preferred in Norway $^{17}$ and North America. ${ }^{22}$

Amoxicillin is the antibiotic of choice for the management of dental infections followed by amoxicillin in combination with clavulanic acid according to the current ${ }^{6,22}$ recommendations. The latter was administered for 51 patients compared to three patients who were prescribed only amoxicillin. This finding also showed the lack of knowledge or carelessness among Lebanese dentists with respect to the emergence of microbial resistance and the occurrence of antibiotic allergies. In contrast, amoxicillin alone is the antibiotic of choice for dentists in countries in the same geographical area, the Middle East. ${ }^{23}$ The difference could be explained by the absence of guidelines endorsed by health authorities in Lebanon as opposed to other countries.

The current recommendations with respect to dosage and duration of therapeutic amoxicillin with or without clavulanic acid ( 2 gm per day for 5-7 days) were in general well respected ${ }^{6}$ except in combination with metronidazole: Only $1 \mathrm{gm}$ of amoxicillin was given for one patient.

The second choice therapeutic antibiotic for the Lebanese dentists, a combination of a macrolide (spiramycin) and metronidazole was administered to $26.60 \%$ of the patients and was similar to the findings of a previous study. ${ }^{9}$ This antibiotic combination is endorsed by the «Agence Française de sécurité sanitaire des produits de santé» (Affssaps). ${ }^{6}$ According to the AHA and the BSC guidelines, the association of antibiotics should be reserved for patients with severe infection, and the administration of only one antibiotic is recommended for dental infections. According to the current recommendations $1.5 \mathrm{gm}$ of metronidazole is prescribed daily for 7 days. ${ }^{6}$ In this study, metronidazole was not properly administered in 
association with amoxicillin. Moreover, unusually high dosages of spiramycin up to $4.5 \mathrm{gm} /$ day in association with metronidazole were given to two patients instead of $2.8 \mathrm{~g}$ as usually recommended. ${ }^{6}$ The simultaneous prescription of two second choice antibiotics would be preferable to the prescription of combined antibiotics in an attempt to reduce the incidence of inadequate dosages observed with the prescription of combined antibiotics because in the first instance, the dentist controls the dosage of each antibiotic.

The misuse of antibiotics was also reported in other studies, which raised concerns related to the lack of recommendations. ${ }^{7,17,19}$

According to Ellison, ${ }^{24}$ the antibiotherapy can be discontinued if clinical signs and symptoms related to the presence of infection subside within 3 days. The AAE 22 recommended a loading antibiotic double dose which would result in a higher antibiotic concentration in the affected areas and a higher bacteriostatic and bactericidal effectiveness. Therefore, the duration of the antibiotherapy could be shortened resulting in a diminished occurrence of microbial resistance and antibiotic allergies.

\section{CONCLUSION}

The present study showed that Lebanese dentists often inappropriately prescribed antibiotics for prophylactic or therapeutic purposes during the management of patients with a dentoalveolar abscess. The medical condition of the patient was a factor predisposing to the prescription of antibiotics. The presence of pain or swelling favored the prescription of antibiotics. The dentists either ignored or were not familiar with the current antibiotic prescription regimens.

\section{ACKNOWLEDGMENT}

Authors would like to thank Hanine Bou Antoun for her help in statistical analysis. 


\section{APPENDIX}

\section{Standardized Form}

Patient Name:

Sex : $\square \mathrm{M}$

Date of Birth:

Date of admission:

Description of the case:

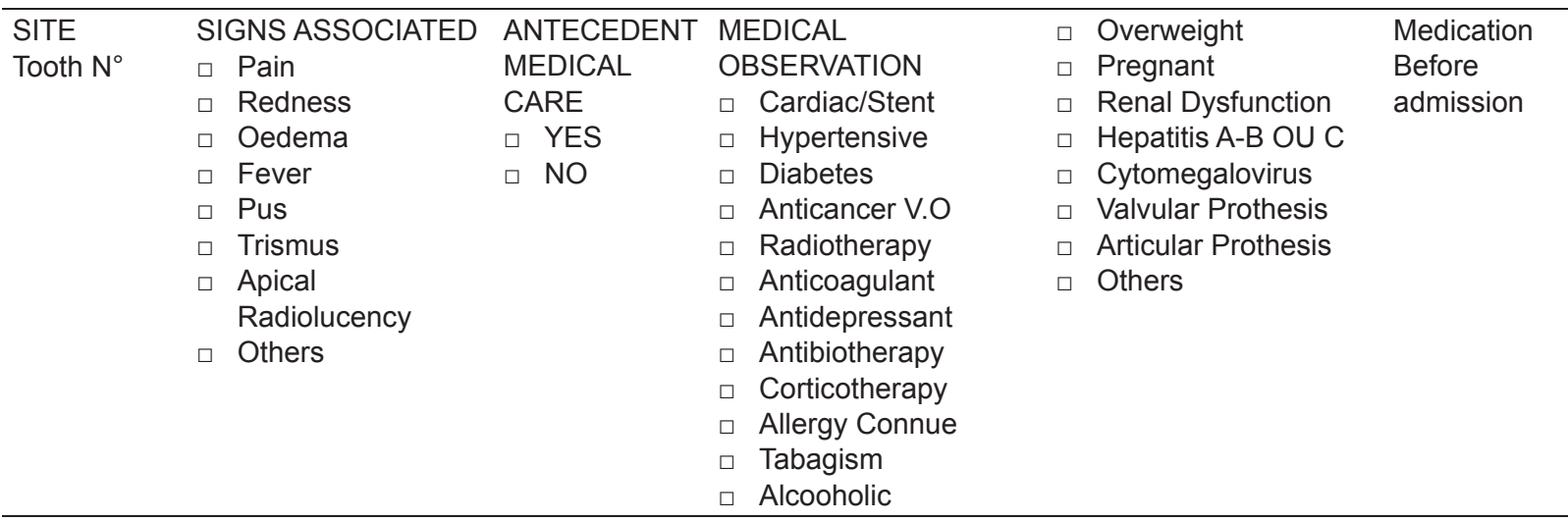

Tel $\mathbf{N}^{\circ}$ :

MEDICAL CARE: Drainage, extraction, endodontic treatment Motif of consultation:

ANTIBIOTICS PRESCRIBED PROPHYLACTIC CURATIVE

\begin{tabular}{|l|l|l|l|l|l|}
\hline Name & Before Care & After Care & Dose & Frequency & Duration \\
\hline & & & & & \\
\hline & & & & & \\
\hline
\end{tabular}

CLINICAL DIAGNOSIS: NECROSIS, PURULENT PULPITIS, PERICORONARITIS, CHRONIC ABCES, CELLULITIS 1-ACUTE ABSCESS:

\begin{tabular}{|l|l|l|}
\hline CELLULITIS & 1st day of consultation & 3rd or 4th day after consultation \\
\hline Estimation Of the Swelling & Diffuse/collected & Absence from 70\% to $80 \%$ or none of the swelling \\
\hline Pus Collection & Diffuse/collected & Diffuse/collected \\
\hline $\begin{array}{l}\text { Swelling in the sulcus of the vestibule } \\
\text { (surelevation) }\end{array}$ & Yes/No & Persistant/non persistant \\
\hline
\end{tabular}

\begin{tabular}{|l|l|l|}
\hline PERICORONARITIS & 1ST day of consultation & 3rd or 4th day after consultation \\
\hline Redness around the tooth & Yes/No & Persistant/non persistant \\
\hline Pain (Estimated by the patient) & Intense/moderate/low & Intense/moderate/low \\
\hline Tismus & Yes/No & Persistant/accentuated/amelioration \\
\hline
\end{tabular}

\section{2-CHRONIC ABSCESS}

\begin{tabular}{|l|l|l|}
\hline \multicolumn{2}{|c|}{ 1st Day } & 3rd or 4th day after consultation \\
\hline CLINICAL SIGNS & & CLINICAL SIGNS \\
\hline Pain at percussion & Yes/No & Yes/No/amelioration \\
\hline Painfull Palpation of the sulcus of the vestibule & Yes/No & Yes/No/amelioration \\
\hline Swelling of the sulcus of the vestibule & Yes/No & Yes/No/amelioration \\
\hline Presence of intra oral fistula & Yes/No & Yes/No/amelioration \\
\hline
\end{tabular}




\section{REFERENCES}

1. Fine D, Hammond B, Loesche W. Clinical use of antibiotics in dental practice. Int J Antimicrob Agents 1998 Feb;9(4):235-238.

2. American Academy of Pediatric Dentistry. Guideline on antibiotic prophylaxis for dental patients at risk for infection. Available from: http://www.aapd.org/media/policies_ guidelines/g_antibioticprophylaxis.pdf.

3. Wynn RL, Bergmann SA. Antibiotics and their use in the treatment of orofacial infections, part I. Gen Dent 1994 SepOct;42(5):398, 400, 402.

4. ADA Council on Scientific Affairs. Antibiotic use in dentistry. J Am Dent Assoc 1997 May;128(5):648.

5. Sroussi HY, Prabhu AR, Epstein JB, American Heart Association, British Society for Antimicrobial Chemotherapy, British Cardiac Society. Which antibiotic prophylaxis guidelines for infective endocarditis should Canadian dentists follow? J Can Dent Assoc 2007 Jun;73(5):401-405.

6. Agence Française De Sécurité Sanitaire Des Produits De Santé AFSSAPS. Prescription of antibiotics for oral and dental care. Med Mal Infect 2012 May;42(5):193-202.

7. Palmer NAO, Pealing R, Ireland RS, Martin MV. A study of therapeutic antibiotic prescribing in National Health Service general dental practice in England. Br Dent J 2000 May;188(10):554-558.

8. Salako NO, Rotimi VO, Adib SM, Al-Mutawa S. Pattern of antibiotic prescription in the management of oral diseases among dentists in Kuwait. J Dent 2004 Sep;32(7):503-509.

9. Al-Haroni M, Skaug N. Incidence of antibiotic prescribing in dental practice in Norway and its contribution to national consumption. J Antimicrob Chemother 2007 Jun;59(6): 1161-1166.

10. Poveda-Roda R, BagánJV,Sanchis-Bielsa JM,Carbonell-PastorE. Antibiotic use in dental practice. A review. Med Oral Pathol Oral Cir Buccal 2007 May;12(3):186-192.

11. Tulip DE, Palmer NAO. A retrospective investigation of the clinical management of patients attending an out of hours dental clinic in Merseyside under the new NHS dental contract. Br Dent J 2008 Dec;205(12):659-664.

12. McCabe WR, Jackson GG. Gram-negative bacteremia. I. Etiology and ecology. Arch Int Med 1962;110(6)845-847.
13. Martin MV, Longman LP, Palmer NAO. Adult antimicrobial prescribing in primary dental care for general dental practitioners. FGDP. London: The Royal College of Surgeons of England; 2000.

14. Centre for Clinical Practice at NICE (UK). Prophylaxis against infective endocarditis: antimicrobial prophylaxis against infective endocarditis in adults and children undergoing interventional procedures. London: National Institute for Health and Clinical Excellence (UK); 2008.

15. Chate RA, White S, Hale LR, Howat AP, Bottomley J, BarnetLamb J, Lindsay J, Davies TI, Heath JM. The impact of clinical audit on antibiotic prescribing in general dental practice. $\mathrm{Br}$ Dent J 2006 Nov;201(10):635-641.

16. Dailey YM, Martin MV. Are antibiotics being used appropriately for emergency dental treatment? Br Dent J 2001 Oct;191(7):391-393.

17. Mainjot A, D'Hoore W, Vanheusden A, Van Nieuwenhuysen JP. Antibiotic prescribing in dental practice in Belgium. Int Endod J 2009 Dec;42(12):1112-1117.

18. Thomas DW, Satterthwaite J, Absi EG, Lewis MA, Shepherd JP. Antibiotic prescription for acute dental conditions in the primary care setting. Br Dent J 1996 Dec 7-21;181(11-12):401-404.

19. Faculty of General Dental Practice (UK). Adult antimicrobial prescribing in primary dental care for general dental practitioners. 2nd ed. London: FGDP (UK); 2012.

20. American Association of Endodontists. Endodontic diagnosis. 2013 Fall. Available from: http://www.aae.org/colleagues.

21. Mata E, Koren LZ, Morse DR, Sinai IH. Prophylactic use of penicillin $\mathrm{V}$ in teeth with necrotic pulps and asymptomatic periapical radiolucencies. Oral Surg Oral Med Pathol 1985 Aug;60(2):201-207.

22. American Association of Endodontists. Use and abuse of antibiotics. 2012 Winter. Available from: http://www.aae. org/colleagues.

23. Gaballah K, Adel A, Bahmani A, Salami A, Hassan M. The knowledge and attitude of practicing dentists towards the antibiotic prescription: a regional study. BJPR 2014;4(16): 2006-2018.

24. Ellison SJ. An outcome audit of three day antimicrobial prescribing for the acute dentoalveolar abscess. Br Dent J 2011 Dec;211(12):591-594. 